\title{
Properties WORTH and WORTHH*, $(1+\delta)$ Embeddings in Banach Spaces with 1-Unconditional Basis and wFPP
}

\author{
Helga Fetter and Berta Gamboa de Buen \\ Centro de Investigación en Matemáticas (CIMAT), Apdo. Postal 402, 36000 Guanajuato, GTO, Mexico
}

Correspondence should be addressed to Helga Fetter, fetter@cimat.mx

Received 24 September 2009; Accepted 3 November 2009

Academic Editor: Mohamed A. Khamsi

Copyright (C) 2010 H. Fetter and B. Gamboa de Buen. This is an open access article distributed under the Creative Commons Attribution License, which permits unrestricted use, distribution, and reproduction in any medium, provided the original work is properly cited.

We will use García-Falset and Lloréns Fuster's paper on the AMC-property to prove that a Banach space $X$ that $(1+\delta)$ embeds in a subspace $X_{\delta}$ of a Banach space $Y$ with a 1-unconditional basis has the property AMC and thus the weak fixed point property. We will apply this to some results by Cowell and Kalton to prove that every reflexive real Banach space with the property WORTH and its dual have the FPP and that a real Banach space $X$ such that $B_{X^{*}}$ is $w^{*}$ sequentially compact and $X^{*}$ has WORTH* has the $w F P P$.

\section{Introduction}

In 1988 Sims [1] introduced the notion of weak orthogonality (WORTH) and asked whether spaces with WORTH have the weak fixed point property (wFPP). Since then several partial answers have been given. For instance, in 1993 García-Falset [2] proved that if $X$ is uniformly nonsquare and has WORTH then it has the wFPP, although Mazcuñán Navarro in her doctoral dissertation [3] showed that uniform nonsquareness is enough. In this work she also showed that WORTH plus 2-UNC implies the wFPP. In both of these cases the space $X$ turns out to be reflexive. In 1994 Sims [4] himself proved that WORTH plus $\varepsilon_{0}$-inquadrate in every direction for some $\varepsilon_{0}<2$ implies the wFPP and in 2003 Dalby [5] showed that if $X^{*}$ has WORTH $^{*}$ and is $\varepsilon_{0}$-inquadrate in every direction for some $\varepsilon_{0}<2$, then $X$ has the wFPP.

Recently in 2008 Cowell and Kalton [6] studied properties $a u$ and $a u^{*}$ in a Banach space $X$, where $a u$ coincides with WORTH if $X$ is separable and $a u^{*}$ in $X$ coincides with WORTH $^{*}$ in $X^{*}$ if $X$ is a separable Banach space. Among other things they proved that a real Banach space with $a u^{*}$ embeds almost isometrically in a space with a shrinking 1unconditional basis and observed that $a u$ and $a u^{*}$ are equivalent if $X$ is reflexive.

We proved, using property AMC shown by García-Falset and Lloréns Fuster [7] to imply the wFPP, that spaces that $(1+\delta)$ embed in a space with a 1-unconditional basis have 
the wFPP. Combining this with Cowell and Kalton's results we were able to show that a reflexive real Banach space with WORTH and its dual both have FPP, giving a partial answer to Sims' question. We also showed that a separable space $X$ such that $X^{*}$ has WORTH* and $B_{X^{*}}$ is $w^{*}$ sequentially compact has the wFPP.

\section{Notations and Definitions}

Let $\left(X,\|\cdot\|_{X}\right)$ be a real Banach space and $K$ a closed nonempty bounded convex subset of $X$.

Definition 2.1. If $x \in X$, we define

$$
R(x, K)=\sup \left\{\|x-z\|_{X}: z \in K\right\}
$$

If $x, y \in K$ the set of quasi-midpoints of $x$ and $y$ in $K$ is given by

$$
M(x, y)=\left\{z \in K: \max \left\{\|z-x\|_{X},\|z-y\|_{X} \leq \frac{1}{2}\|x-y\|_{X}\right\}\right\}
$$

Definition 2.2. $[X]$ is the quotient space $l_{\infty}(X) / c_{0}(X)$ endowed with the norm $\|\tilde{z}\|=$ $\lim \sup _{n}\left\|z_{n}\right\|_{X}$ where $\tilde{z}$ is the equivalence class of $\left(z_{n}\right)$ in $l_{\infty}(X)$, which we also will denote by $\left[z_{n}\right]$. For $x \in X$ we will also denote by $x$ the equivalence class $[(x, x, x, \ldots)]$ in [X]. If $K$ is as above, let $\tilde{K}=\left\{\left[z_{n}\right] \in[X]: z_{n} \in K, n=1,2, \ldots\right\}$. If $Y$ is a Banach space and $T_{n}: X \rightarrow Y$ for $n=1,2, \ldots$, we define $\left[T_{n}\right]=\widetilde{T}, \tilde{T}:[X] \rightarrow[Y]$ by

$$
\tilde{T}\left(\left[z_{n}\right]\right)=\left[T_{n} z_{n}\right] .
$$

If $T_{n}=T$ for $n=1,2, \ldots$ we denote $\tilde{T}$ by $T$.

It is known that $\widetilde{K}$ is also closed bounded and convex in $[X]$ and that $\|\widetilde{T}\|=$ $\lim \sup _{n}\left\|T_{n}\right\|$.

Definition 2.3. Let $\mathcal{S}(\mathbb{N})$ be the set of strictly increasing sequences of natural numbers and $K$ a nonempty bounded convex subset of a Banach space $X$. A sequence $\left(x_{n}\right)$ in $K$ is called equilateral in $K$, if for every $\beta, \gamma \in \mathcal{S}(\mathbb{N})$ such that $\beta(n) \neq \gamma(n)$ for every $n \in \mathbb{N}$, the following equality holds in $[X]$. If $\widetilde{x_{\beta}}=\left[x_{\beta(n)}\right]$ and $\widetilde{x_{\gamma}}=\left[x_{\gamma(n)}\right]$, then

$$
\left\|\widetilde{x_{\beta}}\right\|=\left\|\widetilde{x_{r}}\right\|=\left\|\widetilde{x_{\beta}}-\widetilde{x_{r}}\right\|=D\left(x_{n}\right)=\operatorname{diam}(K),
$$

where $D\left(x_{n}\right)=\lim \sup _{n}\left(\lim \sup _{m}\left\|x_{n}-x_{m}\right\|_{X}\right)$.

It is easy to see that if $\left(x_{n}\right)$ is equilateral in $K$, and $\beta, \gamma \in \mathcal{S}(\mathbb{N})$ are as above, then

$$
\left\|\widetilde{x_{\beta}}-\widetilde{x_{\gamma}}\right\|=\lim _{n}\left\|x_{\beta(n)}-x_{\gamma(n)}\right\|_{X}=\lim _{n}\left\|x_{\beta(n)}\right\|_{X}=\lim _{n}\left\|x_{\gamma(n)}\right\|_{X} .
$$

Now we define the property which interests us in this paper, it was given by GarcíaFalset and Lloréns Fuster in 1990 [7]. 
Definition 2.4. A bounded closed convex subset $K$ of a Banach space $\left(X,\|\cdot\|_{X}\right)$ with $0 \in K$ has the AMC property, if for every weakly null sequence $\left(x_{n}\right)$ which is equilateral in $K$, there exist $\rho \in(0,1), x \in K, \beta, \gamma \in \mathcal{S}(\mathbb{N})$ with $\beta(n) \neq \gamma(n)$ for every $n \in \mathbb{N}$, such that the set

$$
M_{\rho}\left(\left(x_{n}\right), \beta, \gamma\right)=M\left(\widetilde{x_{\beta}}, \widetilde{x_{\gamma}}\right) \cap\left\{\left[z_{n}\right]: d\left(\left[z_{n}\right], K\right) \leq \rho \operatorname{diam}(K)\right\}
$$

is nonempty and $R\left(x, M_{\rho}\left(\left(x_{n}\right), \beta, \gamma\right)\right)<\operatorname{diam}(K) . X$ is said to have AMC if every weakly compact nonempty subset $K$ of $X$ with $0 \in K$ has the AMC property.

\section{Embeddings into Spaces with 1-Unconditional Basis and the wFPP}

Lin in [8] showed that if $X$ has an unconditional basis $\left(e_{n}\right)$ with unconditional constant $\lambda<$ $\left(33^{1 / 2}-3\right) / 2$, then $X$ has the wFPP. García-Falset and Lloréns Fuster proved that in fact under these conditions $X$ has the AMC property which in turn implies the wFPP. We will follow the proof of this closely to establish the next theorem.

Theorem 3.1. Let $\left(X,\|\cdot\|_{X}\right)$ be a Banach space and suppose that there exists a Banach space $\left(Y,\|\cdot\|_{Y}\right)$ with a 1-unconditional basis $\left(e_{n}\right)$ and a subspace $X_{\delta}$ of $Y$ such that $d\left(X, X_{\delta}\right)<(1+\delta)$ where $\delta<(\sqrt{13}-3) / 2$. Then $X$ has AMC and thus the wFPP.

Proof. Let $S: X \rightarrow X_{\delta}$ be an isomorphism with $\|S\| \leq 1+\delta$ and $\left\|S^{-1}\right\| \leq 1$. Let $K \subset X$ be a nonempty weakly compact convex subset of $X$ with $0 \in K$ and diam $(K)=1$. We will show that $K$ has the AMC property.

Let $\left(x_{n}\right)$ be a weakly null equilateral sequence in $K$ and let $K_{\delta}=S(K)$. Then $K_{\delta}$ is weakly compact and $\left(S x_{n}\right)$ is weakly null in $Y$. Hence there exists a sequence $\beta \in \mathcal{S}(\mathbb{N})$ and projections with respect to the basis $\left(e_{n}\right)$ in $Y$ with

(a) $P_{n}: Y \rightarrow s p\left(e_{m_{n}}, e_{m_{n}+1}, \ldots, e_{r_{n}}\right)$ where $m_{n} \leq r_{n}<m_{n+1}$,

(b) $\lim _{n}\left\|P_{n} y\right\|_{Y}=0$ for all $y \in Y$,

(c) $\lim _{n}\left\|S x_{\beta(n)}-P_{n} S x_{\beta(n)}\right\|_{Y}=0$.

Let $\gamma \in \mathcal{S}(\mathbb{N})$ be given by $\gamma(n)=\beta(n+1)$. Then clearly $\beta(n) \neq \gamma(n)$ for every $n \in \mathbb{N}$. Let $\widetilde{P}, \widetilde{Q}:[Y] \rightarrow[Y]$ be given by $\widetilde{P}=\left[P_{n}\right]$ and $\widetilde{Q}=\left[P_{n+1}\right]$ and let $\widetilde{S}:[X] \rightarrow[Y]$ be $\widetilde{S}=[(S, S, \ldots)]$. Recall that we will write $S$ instead of $\widetilde{S}$. By (a), (b), and (c) and since $\left(x_{n}\right)$ is equilateral we have that

(1) $\left\|S \tilde{x}_{\beta}\right\| \leq 1+\delta,\left\|S \tilde{x}_{\gamma}\right\| \leq 1+\delta$ and $\left\|S \tilde{x}_{\beta}-S \tilde{x}_{\gamma}\right\| \leq 1+\delta$,

(2) $\tilde{P} S \tilde{x}_{\beta}=S \tilde{x}_{\beta}, \widetilde{Q} S \tilde{x}_{\gamma}=S x_{\gamma}$,

(3) $\tilde{Q} S \tilde{x}_{\beta}=0, \tilde{P} S \tilde{x}_{\gamma}=0$,

(4) for all $\tilde{y} \in[Y], \widetilde{P} \tilde{y}=\tilde{Q} \tilde{y}=0$.

Therefore, since $\left(e_{n}\right)$ is 1-unconditional

$$
\left\|S \tilde{x}_{\beta}+S \tilde{x}_{\gamma}\right\|=\left\|\tilde{Q} S \tilde{x}_{\beta}+\tilde{P} S \tilde{x}_{\gamma}\right\|=\left\|\tilde{Q} S \tilde{x}_{\beta}-\tilde{P} S \tilde{x}_{\gamma}\right\|=\left\|S \tilde{x}_{\beta}-S \tilde{x}_{\gamma}\right\| \leq 1+\delta
$$


Thus $\left\|\tilde{x}_{\beta}+\tilde{x}_{\gamma}\right\|=\left\|S^{-1} S\left(\tilde{x}_{\beta}+\tilde{x}_{\gamma}\right)\right\| \leq 1+\delta$. Since by hypothesis $\left\|\tilde{x}_{\beta}-\tilde{x}_{\gamma}\right\| \leq 1$, we obtain that $\left(\tilde{x}_{\beta}+\right.$ $\left.\tilde{x}_{\gamma}\right) / 2 \in M_{\rho}\left(\left(x_{n}\right), \beta, \gamma\right)$ if $\delta<1$ and $\rho=(1+\delta) / 2$. Next we will show that $R\left(0, M_{\rho}\left(\left(x_{n}\right), \beta, \gamma\right)\right)<$ 1.

To this effect let $\tilde{w}=\left[w_{n}\right] \in M_{\rho}\left(\left(x_{n}\right), \beta, \gamma\right)$. Define $\tilde{u}=(\tilde{P}+\tilde{Q}) S \tilde{w}$ and $\tilde{r}=(I-(\tilde{P}+$ $\tilde{Q})) S \widetilde{w}$. Then for every $x \in K$

$$
2 \tilde{u}=(\tilde{u}+\tilde{r}-S x)+(\tilde{u}-\tilde{r}+S x) .
$$

By the unconditionality of $\left(e_{n}\right)$ and since by (4) we have that $\widetilde{P} S x=\widetilde{Q} S x=0$,

$$
\begin{aligned}
\|\tilde{u}-\tilde{r}+S x\| & =\|(\widetilde{P}+\tilde{Q})(S \tilde{w}+S x)-(I-(\tilde{P}+\tilde{Q}))(S \tilde{w}-S x)\| \\
& =\|(\widetilde{P}+\widetilde{Q})(S \tilde{w}+S x)+(I-(\widetilde{P}+\tilde{Q}))(S \tilde{w}-S x)\|=\|\tilde{u}+\tilde{r}-S x\| .
\end{aligned}
$$

Therefore

$$
2\|\tilde{u}\| \leq 2\|\tilde{u}+\tilde{r}-S x\|=2\|S \tilde{w}-S x\|
$$

Now let $v \in K$ be such that $\|\tilde{w}-v\| \leq \rho$. Such an element exists since $\tilde{w} \in M_{\rho}\left(\left(x_{n}, \beta, \gamma\right)\right)$. Recalling that $\rho=(1+\delta) / 2$, we obtain that

$$
2\|\tilde{u}\| \leq 2\|S \widetilde{w}-S v\| \leq(1+\delta)^{2} .
$$

Using again that $\widetilde{w} \in M_{\rho}\left(\left(x_{n}, \beta, \gamma\right)\right)$, we get

$$
\begin{aligned}
2\|\tilde{w}\| & \leq\left\|\widetilde{w}+\frac{\widetilde{x_{\beta}}+\widetilde{x_{\gamma}}}{2}\right\|+\left\|\frac{\widetilde{w}-\widetilde{x_{\beta}}}{2}\right\|+\left\|\frac{\widetilde{w}-\widetilde{x_{\gamma}}}{2}\right\| \\
& \leq\left\|\widetilde{w}+\frac{\widetilde{x_{\beta}}+\widetilde{x_{\gamma}}}{2}\right\|+\frac{1}{2}
\end{aligned}
$$

or equivalently

$$
\left\|\widetilde{w}+\frac{\widetilde{x_{\beta}}+\widetilde{x_{\gamma}}}{2}\right\| \geq 2\|\tilde{w}\|-\frac{1}{2} .
$$

On the other hand, since by (2) $\widetilde{P} S x_{\beta}=S x_{\beta}$, we have

$$
\begin{aligned}
\left\|S \tilde{w}+S \widetilde{x_{\beta}}-2 \tilde{P} S \tilde{w}\right\| & =\left\|S \tilde{w}+\tilde{P} S \widetilde{x_{\beta}}-2 \tilde{P} S \tilde{w}\right\|=\left\|(I-\tilde{P}) S \tilde{w}+\widetilde{P}\left(S \widetilde{x_{\beta}}-S \widetilde{w}\right)\right\| \\
& =\left\|(I-\tilde{P}) S \tilde{w}-\tilde{P}\left(S \widetilde{x_{\beta}}-S \widetilde{w}\right)\right\|=\left\|S \widetilde{w}-\tilde{P} S \widetilde{x_{\beta}}\right\|=\left\|S \widetilde{w}-S \widetilde{x_{\beta}}\right\| \\
& \leq(1+\delta)\left\|\widetilde{w}-\widetilde{x_{\beta}}\right\| \leq \frac{1+\delta}{2} .
\end{aligned}
$$


Similarly

$$
\left\|S \tilde{w}+S \widetilde{x_{\gamma}}-2 \tilde{Q} S \tilde{w}\right\| \leq \frac{1+\delta}{2} .
$$

By (3.8) and (3.9), and (3.5) we obtain

$$
\begin{aligned}
2\left\|S \tilde{w}+\frac{S \widetilde{x_{\beta}}+S \widetilde{x_{\gamma}}}{2}\right\| & \leq(1+\delta)+2\|(\tilde{P}+\tilde{Q}) S \tilde{w}\|=(1+\delta)+2\|\tilde{u}\| \\
& \leq(1+\delta)+(1+\delta)^{2} .
\end{aligned}
$$

Hence

$$
\left\|\tilde{w}+\frac{\widetilde{x_{\beta}}+\widetilde{x_{\delta}}}{2}\right\| \leq \frac{(1+\delta)(2+\delta)}{2} .
$$

Finally, from (3.7) and (3.11) we have $2\|\widetilde{w}\|-1 / 2 \leq(1+\delta)(2+\delta) / 2$ and

$$
\|\tilde{w}\| \leq \frac{\delta^{2}+3 \delta+3}{4}
$$

Therefore, if $\delta<(\sqrt{13}-3) / 2$ we conclude that $R\left(0, M_{\rho}\left(\left(x_{n}\right), \beta, \gamma\right)\right)<1$ and thus $X$ has the AMC property.

Remark 3.2. It is evident that if the space $Y$ has a $(1+\lambda)$ unconditional basis, if $\lambda$ is small enough, the above result remains true for some $\delta$.

\section{Some Consequences}

There has always been the conjecture that a space with property WORTH has the wFPP. We show here that this is correct as long as $X$ is reflexive. We also show that property WORTH ${ }^{*}$ in $X^{*}$ implies the wFPP in Banach spaces $X$ so that $B_{X^{*}}$ is $w^{*}$ sequentially compact and that WORTH together with WABS implies the wFPP as well. All these results are consequences of some theorems by Cowell and Kalton [6]. First we need to recall some definitions.

Definition 4.1. A Banach space $X$ has the WORTH property if for every weakly null sequence $\left(x_{n}\right) \subset X$ and every $x \in X$, the following equality holds:

$$
\lim _{n}\left(\left\|x_{n}-x\right\|-\left\|x_{n}+x\right\|\right)=0
$$

This definition was given by Sims in [1]. The next definition was stated by Dalby [5]. 
Definition 4.2. A Banach space $X^{*}$ has the $\mathrm{WORTH}^{*}$ property if for every weak* null sequence $\left(x_{n}^{*}\right) \subset X^{*}$ and every $x^{*} \in X^{*}$, the following equality holds:

$$
\lim _{n}\left(\left\|x_{n}^{*}-x^{*}\right\|-\left\|x_{n}^{*}+x^{*}\right\|\right)=0
$$

[6].

If $X$ is separable and $X^{*}$ has WORTH* ${ }^{*}$, this coincides with the property $a u^{*}$ defined in

Definition 4.3. A Banach space $X$ has the Weak Alternating Banach-Saks (WABS) property if every bounded sequence $\left(x_{n}\right)$ in $X$ has a convex block sequence $\left(y_{n}\right)$ such that

$$
\lim _{n} \sup _{r_{1}<r_{2}<\cdots<r_{n}}\left\|\frac{1}{n} \sum_{j=1}^{n}(-1)^{j} y_{r_{j}}\right\|=0 .
$$

Cowell and Kalton in [6] proved the following three results.

Theorem 4.4. If $X$ is a separable real Banach space, then $X^{*}$ has the property WORTH* if and only if for any $\delta>0$ there is a Banach space $Y$ with a shrinking 1-unconditional basis and a subspace $X_{\delta}$ of $Y$ such that $d\left(X, X_{\delta}\right)<1+\delta$.

Dalby [5] observed that property WORTH* in a space $X^{*}$ implies property WORTH in $X$ and it follows that if $X$ is reflexive, then both properties are equivalent. From this and another theorem we are not going to mention here, Cowell and Kalton obtained the next theorem.

Theorem 4.5. If $X$ is a separable real reflexive space, then $X$ has property WORTH if and only if for any $\delta>0$ there is a reflexive Banach space $Y$ with a 1-unconditional basis and a subspace $X_{\delta}$ of $Y$ such that $d\left(X, X_{\delta}\right)<1+\delta$.

The third result we are going to use is as follows.

Theorem 4.6. If $X$ is a separable real Banach space, then $X$ has both the properties WORTH and WABS if and only if for any $\delta>0$ there is a Banach space $Y$ with a shrinking 1-unconditional basis and a subspace $X_{\delta}$ of $Y$ such that $d\left(X, X_{\delta}\right)<1+\delta$.

From this and our previous work it follows directly the following:

Theorem 4.7. If $X$ is a real separable space such that either

(I) $X^{*}$ has property WORTH*,

(II) $X$ is reflexive and has property WORTH, or

(III) X has both the properties WORTH and WABS,

then $X$ has the property AMC and thus the wFPP.

It is known that reflexivity implies WABS, and thus (II) implies (III), but we want to include (II) in order to deduce the next corollary. Properties WORTH and WABS are inherited by subspaces, and if $X^{*}$ has property WORTH${ }^{*}$ and $B_{X^{*}}$ is $w^{*}$ sequentially compact, then the dual of any subspace of $X$ also has this property. Hence we have the following result. 
Corollary 4.8. Let $X$ be a real Banach space.

(1) If $X$ is reflexive and has property WORTH, then $X$ and $X^{*}$ both have the FPP.

(2) If $X$ has properties WORTH and WABS, then $X$ has the wFPP.

(3) If $X^{*}$ has WORTH* and $B_{X^{*}}$ is $w^{*}$ sequentially compact, then $X$ has the wFPP.

Proof. If $X$ is a Banach space that satisfies (1), (2), or (3), every separable subspace has the wFPP and hence, since the wFPP is separably determined, $X$ has the wFPP. If $X$ is separable and reflexive and has property WORTH, then it has property WORTH* as well and this implies by definition that $X^{*}$ also has property WORTH. Therefore both have the FPP and hence the result follows for nonseparable reflexive spaces.

\section{Acknowledgment}

This work is partially supported by SEP-CONACYT Grant 102380. It is dedicated to W. A. Kirk.

\section{References}

[1] B. Sims, "Orthogonality and fixed points of nonexpansive maps," in Workshop/Miniconference on Functional Analysis and Optimization (Canberra, 1988), vol. 20 of Proceedings of the Centre for Mathematical Analysis, Australian National University, pp. 178-186, The Australian National University, Canberra, Australia, 1988.

[2] J. García-Falset, "The fixed point property in Banach spaces whose characteristic of uniform convexity is less than 2," Journal of the Australian Mathematical Society. Series A, vol. 54, no. 2, pp. 169-173, 1993.

[3] E. M. Mazcuñán Navarro, Geometría de los espacios de Banach en teoría métrica del punto fijo, Tesis doctoral, Universitat de Valencia, Valencia, Spain, 2003.

[4] B. Sims, "A class of spaces with weak normal structure," Bulletin of the Australian Mathematical Society, vol. 49, no. 3, pp. 523-528, 1994.

[5] T. Dalby, "The effect of the dual on a Banach space and the weak fixed point property," Bulletin of the Australian Mathematical Society, vol. 67, no. 2, pp. 177-185, 2003.

[6] S. R. Cowell and N. J. Kalton, “Asymptotic unconditionality,” http://arxiv.org/abs/0809.2294.

[7] J. García-Falset and E. Lloréns Fuster, "A geometric property of Banach spaces related to the fixed point property," Journal of Mathematical Analysis and Applications, vol. 172, no. 1, pp. 39-52, 1993.

[8] P.-K. Lin, "Unconditional bases and fixed points of nonexpansive mappings," Pacific Journal of Mathematics, vol. 116, no. 1, pp. 69-76, 1985. 